\title{
Outage-Based Optimal Power Control for Generalized Multiuser Fading Channels
}

\author{
John Papandriopoulos, Student Member, IEEE, Jamie Evans, Member, IEEE, and \\ Subhrakanti Dey, Senior Member, IEEE
}

\begin{abstract}
We address the problem of achieving outage probability constraints on the uplink of a code-division multiple-access (CDMA) system employing power control and linear multiuser detection, where we aim to minimize the total expended power. We propose a generalized framework for solving such problems under modest assumptions on the underlying channel fading distribution. Unlike previous work, which dealt with a Rayleigh fast-fading model, we allow each user to have a different fading distribution. We show how this problem can be formed as an optimization over user transmit powers and linear receivers, and, where the problem is feasible, we provide conceptually simple iterative algorithms that find the minimum power solution while achieving outage specifications with equality. We further generalize a mapping from outage probability specifications to average signal-to-interference-ratio constraints that was previously applicable only to Rayleigh-faded channels. This mapping allows us to develop suboptimal, computationally efficient algorithms to solve the original problem. Numerical results are provided that validate the iterative schemes, showing the closeness of the optimal and mapped solutions, even under circumstances where the map does not guarantee that constraints will be achieved.
\end{abstract}

Index Terms-Code division multiple access (CDMA), multiuser detection, Nakagami fading, outage probability, power control.

\section{INTRODUCTION}

A DVANCES in wireless services continually rely on higher bit rates and more stringent quality-of-service (QoS) guarantees from carriers. Consequently, there has been an intense research effort to increase the utilization of the limited wireless spectrum through sophisticated physical-layer techniques, the challenge being to simultaneously constrain the system to deliver on a variety of performance measures such as error rate, delay, and throughput. In recent years, interference-limited systems such as those based on code division multiple access (CDMA) have proliferated, and thus a great deal of research effort has focused on addressing the challenge through power control and, even more recently, with multiuser detection (MUD) techniques.

The aim of power control is to intelligently balance the received powers of all users such that no individual creates

Paper approved by K. K. Leung, the Editor for Wireless Network Access and Performance of the IEEE Communications Society. Manuscript received February 6, 2004; revised May 12, 2005. This work was supported by the Australian Research Council. This paper was presented in part at the IEEE International Conference on Communications, Paris, France, June 2004.

The authors are with the ARC Special Research Centre for Ultra-Broadband Information Networks (CUBIN), Department of Electrical and Electronic Engineering, University of Melbourne, Melbourne VIC 3010, Australia (e-mail: jpap@ee.unimelb.edu.au; jse@ee.unimelb.edu.au; sdey@ee.unimelb.edu.au).

Digital Object Identifier 10.1109/TCOMM.2006.873077 excessive interference to others. This is especially important in CDMA networks, as the well-known near-far effect would otherwise significantly degrade performance. In addition to interference management, there are other benefits from power control, such as prolonging the battery life of mobile devices. Similarly, MUD is employed to improve utilization by exploiting the structure of the multiple-access interference (MAI) in order to reduce it [1]-[3]. Substantial improvements in utilization have been observed by combining power control with MUD in a joint optimization over user transmit powers and corresponding receivers. All of these improvements are noticeable, even though these MUD receiver structures are known to have near-far-resistant properties [4]-[6].

Many of the classic papers on power control [7]-[10] formulate the problem as an eigenvalue problem for nonnegative matrices; a survey of this early work can be found in [11]. Later work has concentrated on iterative and distributed algorithms [12]-[16] that are more amenable to practical implementation. More recently, there has been an interest in applying stochastic algorithms to the problem in order to deal with noise-corrupted measurements that serve as inputs to the problem [17], [18]. All of these papers rely on some form of the signal-to-interference ratio (SIR) measure to quantify QoS requirements. With the exception of [18], they further assume the channel to be quasistationary and thus track the instantaneous channel state. This implies a high computational cost and associated digital processing power penalty in battery powered mobile devices as the algorithm is reevaluated each time the instantaneous fading state of the channel changes.

This study deals with situations when it is not feasible or desirable to follow fast fades directly. Rather than demanding that users achieve a target SIR, we relax the constraints and ask that each user maintains its SIR above some prescribed threshold with high probability: we thus consider constraints on outage probability rather than SIR. It turns out that this is similar to the problem considered in [18], where power allocation is made according to the expected value of the instantaneous SIR. We show the connection by deriving a bound on outage probability in terms of an "average SIR."

The problem of power control with constraints on outage probability was considered recently in [19], where interior point methods are employed to find the solution. In [20], we developed a simple iterative algorithm to minimize total transmit power subject to outage constraints. Both of these papers dealt only with Rayleigh fading. In general, work to date assumes a specific fading model, and analysis inherently follows from that selection. A more general framework is needed, so that the 
system designer may simply plug in a desired fading distribution to cater for different cell landscapes. For example, one may use a Rician model for indoor cells with strong line-of-sight components or a Nakagami- $m$ model for outdoor situations lacking strong specular signal components. In practice, the fading distribution is not often Rayleigh nor Nakagami- $m$ with an integer fading figure [21].

In this paper, we specifically address the problem of jointly optimizing user power and multiuser receivers so that each user's outage probability constraint is met with minimum expended total sum power. We do this with only modest conditions on the associated fading distribution.

To summarize, the main contributions of this paper are given here.

1) A framework for developing conceptually simple iterative algorithms that determine user transmit powers such that their sum is minimized, subject to outage constraints. Modest assumptions are made on the fading distribution allowing the system designer to "plug in" various fading models with ease.

2) An extension to the framework for joint optimization over user transmit powers and linear multiuser receivers.

3) A generic procedure to obtain an upper bound on outage probability. This enables a mapping from outage probability to average SIR constraints, allowing us to reformulate an existing algorithm having SIR constraints to solve the above problems suboptimally. Such an algorithm is also conceptually simple, however, it is less computationally expensive to implement [4], [20].

This paper is organized as follows. In Section II, we introduce the system and fading model and derive an expression for the outage probability. Section III formulates a power control problem where linear receivers are fixed. Here, an iterative algorithm that converges to the optimal solution is introduced and convergence proven. We extend these results to a joint power control and multiuser detection problem in Section IV. In Section $\mathrm{V}$, we derive an upper bound on outage probability relating to a margin of average SIR. Using this relation, we introduce a suboptimal joint power control and multiuser receiver optimization problem. Numerical results are presented in Section VI illustrating the tightness of the derived bounds and a comparison of the optimal and suboptimal iterative algorithms under a Nakagami- $m$ fading distribution. Finally, Section VII contains our concluding remarks.

\section{SYSTEM MODEL}

In this paper, we consider the uplink in a direct-sequence synchronous CDMA (DS-SCDMA) communications system with $K$ users and a processing gain of $N$. We assume a binary phaseshift keying (BPSK) modulation scheme and an $N$-dimensional chip matched filter vector at each receiver $i$. The received signal at each filter input is given by

$$
\mathbf{r}_{i}=\sum_{j=1}^{K} \sqrt{G_{i j} F_{i j} P_{j}} b_{j} \mathbf{s}_{j}+\mathbf{n}_{i}, \quad i=1, \ldots, K
$$

where

$G_{i j} \quad$ positive slowly varying path gain of user $j$ to the assigned base station (BS) of user $i$;

$F_{i j} \quad$ associated fast fading component of the channel;

$P_{j} \quad$ transmit power of user $j$;

$b_{j} \quad$ data bits taking on values of \pm 1 with equal probability;

$\mathbf{s}_{j} \quad$ fixed $N$-dimensional spreading sequence of user $j$ with elements taking values $\pm 1 / \sqrt{N}$;

$\mathbf{n}_{i} \quad$ assumed to be AWGN with zero mean and covariance $\sigma^{2} \mathbf{I}$.

We model the fast time-scale fading terms $F_{i j}$ as nonnegative random variables with $E\left[F_{i j}\right]=1$ for all $i, j$. We assume that, for $i$ fixed, each $F_{i j}$ are indepedent for all $j$. Unlike prior work, we do not necessarily assume that these random variables are identically distributed. This allows interference to be modeled by different fading distributions (e.g., different Nakagami- $m$ fading figures). However, we assume that the cumulative distribution function $(\mathrm{CDF})$ of each $F_{i i}$, denoted $\mathcal{F}_{F_{i i}}(\cdot)$, is continuous and strictly increasing on $\mathbb{R}_{+}$. This is the case for most fading distributions of interest, e.g. Nakagami- $m$. We will denote by $\mathbf{F}_{-i}$ the collection of random variables $\left\{F_{i j}: j \neq i\right\}$.

As with prior work, we assume that the slowly varying path gains $G_{i j}$ are known and fixed (we will choose gains from a lognormal distribution multiplied by a distance dependence loss in our numerical simulations of Section VI). In practice, this assumption implies that all results are valid only over a finite time scale where factors affecting these gains do not vary significantly.

Let $\mathbf{c}_{i}$ denote a unit length vector of linear receiver filter coefficients in the $N$-dimensional Euclidean space for user $i$ at its assigned BS and $\mathbf{C}=\left[\mathbf{c}_{1}, \ldots, \mathbf{c}_{K}\right]$. We will assume $\mathbf{c}_{i}$ is always chosen such that $\left(\mathbf{c}_{i}^{\top} \mathbf{s}_{i}\right)^{2}>0$. The filter output of user $i$ at its assigned $\mathrm{BS}$ is given by

$$
\mathbf{y}_{i}=\mathbf{c}_{i}^{\top} \mathbf{r}_{i}=\sum_{j=1}^{K} \sqrt{G_{i j} F_{i j} P_{j}}\left(\mathbf{c}_{i}^{\top} \mathbf{s}_{j}\right) b_{j}+\tilde{n}_{i}
$$

where $\tilde{n}_{i}=\mathbf{c}_{i}^{\top} \mathbf{n}_{i}$ is $N\left(0, \sigma^{2} \mathbf{c}_{i}^{\top} \mathbf{c}_{i}\right)$.

\section{A. SIR and Outage Probability}

The SIR of the $i$ th user is given by

$$
\operatorname{SIR}_{i}\left(\mathbf{P}, \mathbf{c}_{i}\right)=\frac{G_{i i}\left(\mathbf{c}_{i}^{\top} \mathbf{s}_{i}\right)^{2} F_{i i} P_{i}}{\sum_{j \neq i} G_{i j}\left(\mathbf{c}_{i}^{\top} \mathbf{s}_{j}\right)^{2} F_{i j} P_{j}+\sigma^{2}\left(\mathbf{c}_{i}^{\top} \mathbf{c}_{i}\right)}
$$

where we treat receiver noise as interference and $\mathbf{P}$ represents a vector of all user powers.

The corresponding outage probability of user $i$ is defined as the proportion of time that some positive SIR threshold $\gamma_{i}^{\text {th }}$ is 
not met for sufficient reception at the BS receiver. The outage probability for user $i$ is given by

$$
O_{i}\left(\mathbf{P}, \mathbf{c}_{i}\right)=\operatorname{Pr}\left\{\operatorname{SIR}_{i}\left(\mathbf{P}, \mathbf{c}_{i}\right) \leq \gamma_{i}^{\text {th }}\right\}
$$

An alternative expression for the outage is obtained by writing

$$
\begin{aligned}
O_{i} & =\operatorname{Pr}\left\{F_{i i} \leq \Psi_{i}\right\} \\
& =E\left[\mathbf{1}_{\left\{F_{i i} \leq \Psi_{i}\right\}}\right] \\
& \stackrel{(a)}{=} E\left[E\left[\mathbf{1}_{\left\{F_{i i} \leq \Psi_{i}\right\}} \mid \mathbf{F}_{-i}\right]\right] \\
& =E\left[\operatorname{Pr}\left\{F_{i i} \leq \Psi_{i}\right\} \mid \mathbf{F}_{-i}\right] \\
& \stackrel{(b)}{=} E\left[\mathcal{F}_{F_{i i}}\left(\Psi_{i}\right)\right]
\end{aligned}
$$

where we make use of a standard result on conditional expectation [22, Proposition 4.1] to obtain the equality (a) and the independence of the $F_{i j}$ in (b)

$$
\Psi_{i}=\gamma_{i}^{\mathrm{th}} \frac{\sum_{j \neq i} G_{i j}\left(\mathbf{c}_{i}^{\top} \mathbf{s}_{j}\right)^{2} F_{i j} P_{j}+\sigma^{2}\left(\mathbf{c}_{i}^{\top} \mathbf{c}_{i}\right)}{G_{i i}\left(\mathbf{c}_{i}^{\top} \mathbf{s}_{i}\right)^{2} P_{i}}
$$

and $\mathbf{1}_{A}$ is an indicator function of the event $A$ taking a value of 1 when $A$ is true, and 0 otherwise.

Remark: The outage probability does not directly depend on the instantaneous fast-fading state of the channel; rather, it relies on the statistics of the chosen fading distribution used to model the fast-fading component of the channel.

\section{USER POWER OPtimizATION With OUTAGE CONSTRAints}

Here, we address the problem of optimizing user powers subject to outage constraints. We first consider the situation where linear receivers are fixed. Without a loss of generality, we can omit the fixed linear receiver filter terms $\left(\mathbf{c}_{i}^{\top} \mathbf{s}_{j}\right)^{2}$ from the notation, since we can absorb them into the $G_{i j}$ terms.

\section{A. Problem Definition}

We wish to find each user's transmit power level such that the total power transmitted by all users is minimized while meeting all outage probability constraints. Stating this as a static optimization problem, we have

$$
\begin{aligned}
& \min _{\mathbf{P} \geq \mathbf{0}} \sum_{i=1}^{K} P_{i} \\
& \text { s.t. } \quad O_{i}(\mathbf{P}) \leq \Omega_{i}, \quad i=1, \ldots, K
\end{aligned}
$$

where $\boldsymbol{\Omega}=\left[\Omega_{1}, \ldots, \Omega_{K}\right]$ specifies all users' outage constraints in the range $0<\Omega_{i}<1$ and $O_{i}(\mathbf{P})$ as defined in (3), with a fixed $\mathbf{c}_{i}$.

\section{B. Outage Probability—Monotonicity}

The following general results for outage probability are crucial to the formulation of an algorithm to solve the above optimization problem.

Result 1: $O_{i}(\mathbf{P})$ is strictly decreasing in $P_{i}$ and as $P_{i} \rightarrow \infty$, $O_{i}(\mathbf{P}) \rightarrow 0$; as $P_{i} \rightarrow 0, O_{i}(\mathbf{P}) \rightarrow 1$.

Proof: Assume $P_{i}^{\prime}>P_{i}>0$. Expanding (4) gives

$$
\begin{aligned}
O_{i}(\mathbf{P}) & =E\left[\mathcal{F}_{F_{i i}}\left(\gamma_{i}^{\mathrm{th}} \frac{\sum_{j \neq i} G_{i j} F_{i j} P_{j}+\sigma^{2}}{G_{i i} P_{i}}\right)\right] \\
& =E\left[\mathcal{F}_{F_{i i}}\left(\frac{1}{P_{i}}[\cdot]\right)\right]
\end{aligned}
$$

and

$$
\begin{aligned}
O_{i}\left(\left.\mathbf{P}\right|_{P_{i}=P_{i}^{\prime}}\right) & =E\left[\mathcal{F}_{F_{i i}}\left(\gamma_{i}^{\text {th }} \frac{\sum_{j \neq i} G_{i j} F_{i j} P_{j}+\sigma^{2}}{G_{i i} P_{i}^{\prime}}\right)\right] \\
& =E\left[\mathcal{F}_{F_{i i}}\left(\frac{1}{P_{i}^{\prime}}[\cdot]\right)\right]
\end{aligned}
$$

where $[\cdot]=\left(\gamma_{i}^{\text {th }} / G_{i i}\right)\left(\sum_{j \neq i} G_{i j} F_{i j} P_{j}+\sigma^{2}\right)$.

The inequality

$$
\mathcal{F}_{F_{i i}}\left(\frac{1}{P_{i}}[\cdot]\right)>\mathcal{F}_{F_{i i}}\left(\frac{1}{P_{i}^{\prime}}[\cdot]\right)
$$

follows from the assumed monotonicity property of $\mathcal{F}_{F_{i i}}(\cdot)$. Clearly then

$$
\begin{aligned}
O_{i}(\mathbf{P}) & =E\left[\mathcal{F}_{F_{i i}}\left(\frac{1}{P_{i}}[\cdot]\right)\right] \\
& >E\left[\mathcal{F}_{F_{i i}}\left(\frac{1}{P_{i}^{\prime}}[\cdot]\right)\right] \\
& =O_{i}\left(\left.\mathbf{P}\right|_{P_{i}=P_{i}^{\prime}}\right) .
\end{aligned}
$$

In addition, we note immediately that

$$
\lim _{P_{i} \rightarrow \infty} E\left[\mathcal{F}_{F_{i i}}\left(\frac{1}{P_{i}}[\cdot]\right)\right]=\lim _{x \rightarrow 0} \mathcal{F}_{F_{i i}}(x)=0
$$

and

$$
\lim _{P_{i} \rightarrow 0} E\left[\mathcal{F}_{F_{i i}}\left(\frac{1}{P_{i}}[\cdot]\right)\right]=\lim _{x \rightarrow \infty} \mathcal{F}_{F_{i i}}(x)=1
$$

by definition of the $\operatorname{CDF} \mathcal{F}_{F_{i i}}(\cdot)$.

Result 2: $O_{i}(\mathbf{P})$ is increasing in $P_{j}, j \neq i$. 

gives

Proof: Assume $P_{j}^{\prime}>P_{j}>0$ for any $j \neq i$. Expanding (4)

$$
\begin{aligned}
O_{i}(\mathbf{P}) & =E\left[\mathcal{F}_{F_{i i}}\left(\gamma_{i}^{\text {th }} \frac{\sum_{\substack{q \neq i \\
q \neq j}} G_{i q} F_{i q} P_{q}+G_{i j} F_{i j} P_{j}+\sigma^{2}}{G_{i i} P_{i}}\right)\right] \\
& =E\left[\mathcal{F}_{F_{i i}}\left(\frac{\gamma_{i}^{\text {th }}}{G_{i i} P_{i}}\left[[\cdot]+G_{i j} F_{i j} P_{j}\right]\right)\right]
\end{aligned}
$$

and

$$
\begin{aligned}
O_{i} & \left(\left.\mathbf{P}\right|_{P_{j}=P_{j}^{\prime}}\right) \\
& =E\left[\mathcal{F}_{F_{i i}}\left(\gamma_{i}^{\mathrm{th}} \frac{\sum_{\substack{q \neq i \\
q \neq j}} G_{i q} F_{i q} P_{q}+G_{i j} F_{i j} P_{j}^{\prime}+\sigma^{2}}{G_{i i} P_{i}}\right)\right] \\
& =E\left[\mathcal{F}_{F_{i i}}\left(\frac{\gamma_{i}^{\mathrm{th}}}{G_{i i} P_{i}}\left[[\cdot]+G_{i j} F_{i j} P_{j}^{\prime}\right]\right)\right]
\end{aligned}
$$

where [.] $=\sum_{\substack{q \neq i \\ q \neq j}} G_{i q} F_{i q} P_{q}+\sigma^{2}$.

The inequality

$$
\begin{aligned}
\mathcal{F}_{F_{i i}}\left(\frac{\gamma_{i}^{\text {th }}}{G_{i i} P_{i}}\left[[\cdot]+G_{i j} F_{i j} P_{j}\right]\right) & \\
& \leq \mathcal{F}_{F_{i i}}\left(\frac{\gamma_{i}^{\text {th }}}{G_{i i} P_{i}}\left[[\cdot]+G_{i j} F_{i j} P_{j}^{\prime}\right]\right)
\end{aligned}
$$

follows from the assumed monotonicity property of $\mathcal{F}_{F_{i i}}(\cdot)$, noting that equality holds whenever $G_{i j}=0$. Clearly then

$$
\begin{aligned}
O_{i}(\mathbf{P}) & =E\left[\mathcal{F}_{F_{i i}}\left(\frac{\gamma_{i}^{\text {th }}}{G_{i i} P_{i}}\left[[\cdot]+G_{i j} F_{i j} P_{j}\right]\right)\right] \\
& \leq E\left[\mathcal{F}_{F_{i i}}\left(\frac{\gamma_{i}^{\text {th }}}{G_{i i} P_{i}}\left[[\cdot]+G_{i j} F_{i j} P_{j}^{\prime}\right]\right)\right] \\
& =O_{i}\left(\left.\mathbf{P}\right|_{P_{j}=P_{j}^{\prime}}\right) .
\end{aligned}
$$

Result 3: Scaling up all users' powers by the same factor lowers the outage probability, i.e. $O_{i}(\alpha \mathbf{P})<O_{i}(\mathbf{P})$ if $\alpha>1$, for all $i$.

Proof: Since the receiver noise variance $\sigma^{2}$ is nonzero, then

$$
\begin{aligned}
O_{i}(\alpha \mathbf{P}) & =E\left[\mathcal{F}_{F_{i i}}\left(\gamma_{i}^{\text {th }} \frac{\sum_{j \neq i} \alpha G_{i j} F_{i j} P_{j}+\sigma^{2}}{\alpha G_{i i} P_{i}}\right)\right] \\
& <E\left[\mathcal{F}_{F_{i i}}\left(\gamma_{i}^{\text {th }} \frac{\sum_{j \neq i} \alpha G_{i j} F_{i j} P_{j}+\alpha \sigma^{2}}{\alpha G_{i i} P_{i}}\right)\right] \\
& =O_{i}(\mathbf{P}) .
\end{aligned}
$$

\section{Optimal Solution}

Assuming that the feasible set is nonempty, we have the following results relating to the optimal solution.

Lemma 1: The optimal solution $\mathbf{P}^{*}$ will have outage constraints satisfied with equality. That is, $O_{i}\left(\mathbf{P}^{*}\right)=\Omega_{i}$ for all $i$, where we recall that $0<\Omega_{i}<1$.

Proof: Suppose $\mathbf{P}$ is a power vector in the feasible set and that there exists a user with $O_{i}(\mathbf{P})<\Omega_{i}$. Using Results 1 and 2 , we see that we can lower the power of user $i$ while keeping all outage constraints satisfied. This means that there is a feasible set of powers with smaller sum than $\mathbf{P}$ so that $\mathbf{P}$ is clearly not optimal.

Lemma 2: With $P_{j}$ fixed for all $j \neq i$, the equation $O_{i}(\mathbf{P})=$ $\Omega_{i}$ has a unique positive solution $P_{i}=P_{i}^{*}$.

Proof: Immediately from Result 1.

Denote the unique solution of Lemma 2

$$
P_{i}^{*}=I_{i}\left(\mathbf{P}_{-i}\right)
$$

where $\mathbf{P}_{-i}$ are $(K-1)$-length vectors, having the same form as the full-length power vector with the $i$ th element omitted.

The function $I_{i}(\cdot)$ specifies the power required by user $i$ to meet their outage constraint with equality when interfering users have fixed powers $\mathbf{P}_{-i}$. Envisage an algorithm where, starting from some initial power vector, each user independently updates their power to meet their outage constraint-assuming that the other users powers are fixed. This leaves us with a new set of powers which form the starting point for the next iteration. This is an intuitively pleasing algorithm but will it converge to the solution of our optimization problem?

With the above algorithm in mind, define

$$
\mathbf{I}(\mathbf{P})=\left[I_{1}\left(\mathbf{P}_{-1}\right), \ldots, I_{K}\left(\mathbf{P}_{-K}\right)\right] .
$$

We shall refer to (6) as the interference function to maintain consistency with the framework in [23] and other prior work [20].

We propose a new power control algorithm (PCA) having the standard form

$$
\mathbf{P}^{n+1}=\mathbf{I}\left(\mathbf{P}^{n}\right)
$$

where $n$ denotes the iteration step. For convenience, we initialize the algorithm with powers set to the receiver noise level $P_{i}^{0}=\sigma^{2}$ for all $i$, however, any positive value can be chosen in practice. Theorem 1 proposes that (7) is a standard interference function and, as a consequence, the PCA converges to a fixed point. The proof is based on the properties of such functions, which were introduced in [23] and are repeated below.

Definition 1: Interference function $\mathbf{I}(\mathbf{P})$ is standard if, for all $\mathbf{P} \geq 0$, the following properties are satisfied.

- Positivity $\mathbf{I}(\mathbf{P})>0$.

- Monotonicity If $\mathbf{P} \geq \mathbf{P}^{\prime}$, then $\mathbf{I}(\mathbf{P}) \geq \mathbf{I}\left(\mathbf{P}^{\prime}\right)$.

- Scalability For all $\alpha>1, \alpha \mathbf{I}(\mathbf{P})>\mathbf{I}(\alpha \mathbf{P})$.

Theorem 1: $\mathbf{I}(\mathbf{P})$ is a standard interference function. 
Proof: Positivity is immediate from Lemma 2.

We next observe that, for any user $i, I_{i}\left(\mathbf{P}_{-i}\right)$ is defined by $O_{i}\left(\left.\mathbf{P}\right|_{P_{i}=I_{i}\left(\mathbf{P}_{-i}\right)}\right)=\Omega_{i}$, and we will use this observation to prove the monotonicity and scalability properties by contradiction.

First, suppose $\mathbf{P} \geq \mathbf{P}^{\prime}$ and the converse is true: $I_{i}\left(\mathbf{P}_{-i}\right)<$ $I_{i}\left(\mathbf{P}_{-i}^{\prime}\right)$. Then

$$
\begin{aligned}
O_{i}\left(\left.\mathbf{P}\right|_{P_{i}=I_{i}\left(\mathbf{P}_{-i}\right)}\right) & \stackrel{(a)}{\geq} O_{i}\left(\left.\mathbf{P}^{\prime}\right|_{P_{i}^{\prime}=I_{i}\left(\mathbf{P}_{-i}\right)}\right) \\
& \stackrel{(b)}{>} O_{i}\left(\left.\mathbf{P}^{\prime}\right|_{P_{i}^{\prime}=I_{i}\left(\mathbf{P}_{-i}^{\prime}\right)}\right)
\end{aligned}
$$

where $(a)$ follows from Result 2 and $(b)$ follows from Result 1. However, by definition, we have

$$
O_{i}\left(\left.\mathbf{P}\right|_{P_{i}=I_{i}\left(\mathbf{P}_{-i}\right)}\right)=O_{i}\left(\left.\mathbf{P}^{\prime}\right|_{P_{i}^{\prime}=I_{i}\left(\mathbf{P}_{-i}^{\prime}\right)}\right)=\Omega_{i}
$$

and thus $I_{i}\left(\mathbf{P}_{-i}\right) \geq I_{i}\left(\mathbf{P}_{-i}^{\prime}\right)$. Furthermore, this holds for all $i$, and thus we conclude $\mathbf{I}(\mathbf{P}) \geq \mathbf{I}\left(\mathbf{P}^{\prime}\right)$ and (6) satisfies the monotonicity property.

Suppose $\mathbf{P}^{\prime}=\alpha \mathbf{P}$ with $\alpha>1$ and the converse is true: $\alpha I_{i}\left(\mathbf{P}_{-i}\right) \leq I_{i}\left(\alpha \mathbf{P}_{-i}\right)$ or, equivalently, $\alpha I_{i}\left(\mathbf{P}_{-i}\right) \leq I_{i}\left(\mathbf{P}_{-i}^{\prime}\right)$. Then

$$
\begin{aligned}
O_{i}\left(\left.\mathbf{P}^{\prime}\right|_{P_{i}^{\prime}=I_{i}\left(\mathbf{P}_{-i}^{\prime}\right)}\right) & \stackrel{(c)}{\leq} O_{i}\left(\left.\mathbf{P}^{\prime}\right|_{P_{i}^{\prime}=\alpha I_{i}\left(\mathbf{P}_{-i}\right)}\right) \\
& \stackrel{(d)}{<} O_{i}\left(\left.\mathbf{P}\right|_{P_{i}=I_{i}\left(\mathbf{P}_{-i}\right)}\right)
\end{aligned}
$$

where $(c)$ follows from Result 1 and $(d)$ follows from Result 3.

However, by definition, we have

$$
O_{i}\left(\left.\mathbf{P}^{\prime}\right|_{P_{i}^{\prime}=I_{i}\left(\mathbf{P}_{-i}^{\prime}\right)}\right)=O_{i}\left(\left.\mathbf{P}\right|_{P_{i}=I_{i}\left(\mathbf{P}_{-i}\right)}\right)=\Omega_{i}
$$

and thus $\alpha I_{i}\left(\mathbf{P}_{-i}\right)>I_{i}\left(\mathbf{P}_{-i}^{\prime}\right)$. Equivalently, $\alpha I_{i}\left(\mathbf{P}_{-i}\right)>$ $I_{i}\left(\alpha \mathbf{P}_{-i}\right)$. This holds for all $i$, and thus we conclude $\alpha \mathbf{I}(\mathbf{P})>\mathbf{I}(\alpha \mathbf{P})$, and (6) satisfies the scalability property.

Since $\mathbf{I}(\mathbf{P})$ is a standard interference function, the PCA (7) converges to a fixed point regardless of the initial power vector. Furthermore, this point is indeed the optimal solution to the above optimization problem [23, Thm. 2]].

Remark: While the original problem involved solving a coupled system of $K$ nonlinear equations in $K$ unknowns, each step of the proposed algorithm requires the separate solution of $K$ equations, each in one variable. As such, this algorithm lends itself well to parallel computation.

\section{JOINT POWER AND MUD OPTIMIZATION WITH OUTAGE CONSTRAINTS}

Here, we consider the situation where we have a choice over linear receivers and no longer neglect the receiver terms $\left(\mathbf{c}_{i}^{\top} \mathbf{s}_{j}\right)^{2}$.

\section{A. Revised Problem Definition}

We wish to jointly find each user's power $\mathbf{P}=\left[P_{1}, \ldots, P_{K}\right]$ and linear receivers $\mathbf{C}=\left[\mathbf{c}_{1}, \ldots, \mathbf{c}_{K}\right]$ such that the total power transmitted by all users is minimized, while meeting all outage probability constraints. Mathematically, we form the optimization problem

$$
\begin{aligned}
\min _{\mathbf{P} \geq \mathbf{0}, \mathbf{C}} & \sum_{i=1}^{K} P_{i} \\
\text { s.t. } \quad & O_{i}\left(\mathbf{P}, \mathbf{c}_{i}\right) \leq \Omega_{i} \\
& \left\|\mathbf{c}_{i}\right\|_{2}=1, \quad i=1, \ldots, K
\end{aligned}
$$

and is equivalent to

$$
\begin{aligned}
& \min _{\mathbf{P} \geq \mathbf{0}} \sum_{i=1}^{K} P_{i} \\
& \text { s.t. } \min _{\mathbf{c}_{i},\left\|\mathbf{c}_{i}\right\|_{2}=1} O_{i}\left(\mathbf{P}, \mathbf{c}_{i}\right) \leq \Omega_{i}, \quad i=1, \ldots, K .
\end{aligned}
$$

See [4] and [20] for similar refinements.

\section{B. Optimal Solution}

Define a new function $\mathbf{J}(\mathbf{P})=\left[J_{1}\left(\mathbf{P}_{-1}\right), \ldots, J_{K}\left(\mathbf{P}_{-K}\right)\right]$, where each component is given by

$$
J_{i}\left(\mathbf{P}_{-i}\right)=\min _{\mathbf{c}_{i},\left\|\mathbf{c}_{i}\right\|_{2}=1} I_{i}\left(\mathbf{P}_{-i}, \mathbf{c}_{i}\right)
$$

and where $I_{i}\left(\mathbf{P}_{-i}, \mathbf{c}_{i}\right)$ was defined in (5). Mirroring the development in Section III, we now propose a new PCA having the standard form $\mathbf{P}^{n+1}=\mathbf{J}\left(\mathbf{P}^{n}\right)$, where $n$ denotes the iteration step. The algorithm is initialized with powers set to the receiver noise power.

We now present a convergence result for this PCA, which is based heavily on the properties of Definition 1 .

Theorem 2: $\mathbf{J}(\mathbf{P})$ is a standard interference function.

Proof: Recall the positivity result from Theorem 1: $I_{i}\left(\mathbf{P}_{-i}, \mathbf{c}_{i}\right)>0$ for any fixed $\mathbf{c}_{i}$. It follows that (8) is also positive.

Assume $\mathbf{P} \geq \mathbf{P}^{\prime}$ and let $\mathbf{c}_{i}^{*}=\arg \min _{\mathbf{c}_{i},\left\|\mathbf{c}_{i}\right\|=1} I_{i}\left(\mathbf{P}_{-i}, \mathbf{c}_{i}\right)$. We then have

$$
\begin{aligned}
J_{i}\left(\mathbf{P}_{-i}\right) & =\min _{\mathbf{c}_{i},\left\|\mathbf{c}_{i}\right\|=1} I_{i}\left(\mathbf{P}_{-i}, \mathbf{c}_{i}\right) \\
& =I_{i}\left(\mathbf{P}_{-i}, \mathbf{c}_{i}^{*}\right) \\
& \geq I_{i}\left(\mathbf{P}_{-i}^{\prime}, \mathbf{c}_{i}^{*}\right) \\
& \geq \min _{\mathbf{c}_{i},\left|\mathbf{c}_{i}\right|=1} I_{i}\left(\mathbf{P}_{-i}^{\prime}, \mathbf{c}_{i}\right) \\
& =J_{i}\left(\mathbf{P}_{-i}^{\prime}\right)
\end{aligned}
$$

and thus (8) satisfies the monotonicity property. 
To prove scalability, we have

$$
\begin{aligned}
\alpha J_{i}\left(\mathbf{P}_{-i}\right) & =\min _{\mathbf{c}_{i},\left\|\mathbf{c}_{i}\right\|=1} \alpha I_{i}\left(\mathbf{P}_{-i}, \mathbf{c}_{i}\right) \\
& =\alpha I_{i}\left(\mathbf{P}_{-i}, \mathbf{c}_{i}^{*}\right) \\
& >I_{i}\left(\alpha \mathbf{P}_{-i}, \mathbf{c}_{i}^{*}\right) \\
& >\min _{\mathbf{c}_{i},\left\|\mathbf{c}_{i}\right\|=1} I_{i}\left(\alpha \mathbf{P}_{-i}, \mathbf{c}_{i}\right) \\
& =J_{i}\left(\alpha \mathbf{P}_{-i}\right)
\end{aligned}
$$

where $\mathbf{c}_{i}^{*}$ is defined above and we have used the scalability property of $I_{i}(\cdot, \cdot)$ for a fixed $\mathbf{c}_{i}$.

Given that $J_{i}(\cdot, \cdot)$ satisfies the positivity, monotonicity, and scalability properties for any user $i$, we conclude that $\mathbf{J}(\cdot, \cdot)$ is also a standard interference function.

We have shown that $\mathbf{J}(\mathbf{P})$ is a standard interference function and, indeed, this new PCA converges to the optimal fixed point $\mathbf{P}^{*}=\mathbf{J}\left(\mathbf{P}^{*}\right)$. This solution is the minimum power required to meet all user outage constraints, with linear receivers having converged to the minimum outage probability (MOP) receiver [20].

We refer to this algorithm as the MOP-PCA.

\section{BOUNDS ON OUTAGE PROBABILITY}

In our earlier work, we have shown that the outage expression for the Rayleigh fading channel could be mapped to an $a v$ erage SIR threshold, allowing the original problem to be transformed to a more familiar one involving average SIR and average SIR constraints [20]. The resultant algorithm utilized the closed-form MMSE receiver, thus relieving the need for a computationally expensive $N$-dimensional minimization over $\mathbf{c}_{i}$, as is required by the MOP-PCA.

This section develops an upper bound on outage probability in a more general fashion. When the bound applies, the optimization problems above can be solved suboptimally using highly efficient iterative methods, where the optimization over $\mathbf{c}_{i}$ is now known in closed form. Furthermore, large system approximations can also be used to solve the problem in a decentralized manner without iteration [20]. We numerically investigate the closeness of the suboptimal solution in Section VI.

\section{A. Certainty Equivalence Margin}

The certainty-equivalent margin (CEM) was defined in [19] for the noiseless case and further refined to include receiver noise in [20]. The CEM represents a margin of error for average SIR when representing a system by its certainty-equivalent form, with all statistical variation in signal and noise power ignored and replaced with their expected values.

Average SIR, denoted $\overline{\text { SIR }}$, is defined as

$$
\overline{\operatorname{SIR}}_{i}\left(\mathbf{P}, \mathbf{c}_{i}\right)=\frac{G_{i i}\left(\mathbf{c}_{i}^{\top} \mathbf{s}_{i}\right)^{2} P_{i}}{\sum_{j \neq i} G_{i j}\left(\mathbf{c}_{i}^{\top} \mathbf{s}_{j}\right)^{2} P_{j}+\sigma^{2}\left(\mathbf{c}_{i}^{\top} \mathbf{c}_{i}\right)}
$$

while CEM (with noise) is the ratio of the $\overline{\mathrm{SIR}}$ to the SIR threshold

$$
\operatorname{CEM}_{i}^{\sigma}\left(\mathbf{P}, \mathbf{c}_{i}\right)=\frac{1}{\gamma_{i}^{\text {th }}} \frac{G_{i i}\left(\mathbf{c}_{i}^{\top} \mathbf{s}_{i}\right)^{2} P_{i}}{\sum_{j \neq i} G_{i j}\left(\mathbf{c}_{i}^{\top} \mathbf{s}_{j}\right)^{2} P_{j}+\sigma^{2}\left(\mathbf{c}_{i}^{\top} \mathbf{c}_{i}\right)}
$$

To simplify the notation, we will occasionally drop these functional forms and denote the average SIR and CEM as simply $\overline{\operatorname{SIR}}_{i}$ and $\mathrm{CEM}_{i}^{\sigma}$, respectively.

\section{B. Upper Bound on Outage Probability}

Suppose that the fading $\operatorname{CDF} \mathcal{F}_{F_{i i}}(\cdot)$ is concave on $\mathbb{R}_{+}$. Then, by Jensen's inequality, we have

$$
\begin{aligned}
O_{i} & =E\left[\mathcal{F}_{F_{i i}}\left(\Psi_{i}\right)\right] \\
& \leq \mathcal{F}_{F_{i i}}\left(E\left[\Psi_{i}\right]\right) \\
& =\mathcal{F}_{F_{i i}}\left(\gamma_{i}^{\text {th }} \frac{\sum_{j \neq i} G_{i j}\left(\mathbf{c}_{i}^{\top} \mathbf{s}_{j}\right)^{2} E\left[F_{i j}\right] P_{j}+\sigma^{2}\left(\mathbf{c}_{i}^{\top} \mathbf{c}_{i}\right)}{G_{i i}\left(\mathbf{c}_{i}^{\top} \mathbf{s}_{i}\right)^{2} P_{i}}\right) \\
& =\mathcal{F}_{F_{i i}}\left(\frac{1}{\mathrm{CEM}_{i}^{\sigma}}\right) .
\end{aligned}
$$

This bound allows us to map outage constraints to constraints on CEM or average SIR, which are constraints that are much easier to deal with.

\section{Examples}

We now show examples of how this bound may be derived by concentrating on the popular Nakgami- $m$ fading distribution that recovers the Rayleigh distribution when $m=1$.

We choose to focus on this distribution since the Rician is well approximated by Nakgami- $m$ with $m=(\eta+1)^{2} /(2 \eta+1) \geq 1$, where $\eta$ is the Rice factor [24, Sec. 3.3]. In general, however, experimental results suggest that the Nakgami- $m$ distribution gives a better fit to measured data than other alternatives, doing so over a variety of cell landscapes [25].

1) Rayleigh: A received signal having power $y$ with a Rayleigh distributed envelope has a cumulative power distribution

$$
\mathcal{F}_{Y}(y)=1-e^{-y}, \quad y \geq 0
$$

when $E[y]=1$. It is trivial to show that (12) is concave for all $y \geq 0$. Thus, we have the upper bound on outage given by

$$
O_{i} \leq 1-\exp \left(-\frac{1}{\mathrm{CEM}_{i}^{\sigma}}\right)
$$

utilizing (11), where we assume that all users have i.i.d. fading distributions. This is exactly the upper bound found previously via different means [20, eq. (9)]. 
2) Nakgami-m: The received signal power $y$ for a general fading figure $m$ has cumulative power distribution

$$
\mathcal{F}_{Z}(y)=\frac{1}{\Gamma(m)} m^{m} \int_{0}^{y} z^{m-1} e^{-m z} d z, \quad y \geq 0
$$

when $E[y]=1$ and we again assume i.i.d. fading.

We can differentiate twice to yield

$$
\frac{d^{2} \mathcal{F}_{Z}(y)}{d y^{2}}=\frac{1}{\Gamma(m)} m^{m} y^{m-2} e^{-m y}[(m-1)-m y]
$$

Observe that $d^{2} \mathcal{F}_{Z}(y) / d y^{2}<0$ for all $y>0$ provided $m \leq 1$. Thus, the upper bound only applies for $m \leq 1$ as

$$
\begin{aligned}
O_{i} & \leq \mathcal{F}_{Z}\left(\frac{1}{\mathrm{CEM}_{i}^{\sigma}}\right) \\
& =\frac{1}{\Gamma(m)} m^{m} \int_{0}^{\frac{1}{\mathrm{CEM}_{i}^{\sigma}}} z^{m-1} e^{-m z} d z \\
& =\frac{1}{\Gamma(m)} \int_{0}^{\frac{m}{\mathrm{CEM}_{i}^{\sigma}}} t^{m-1} e^{-t} d z \\
& =\Gamma\left(\frac{m}{\mathrm{CEM}_{i}^{\sigma}}, m\right)
\end{aligned}
$$

where

$$
\Gamma(x, a)=\frac{1}{\Gamma(a)} \int_{0}^{x} e^{-t} t^{a-1} d t
$$

is an incomplete Gamma function.

\section{Mapping From Outage to Average SIR Constraints}

Assume the upper bound of (11) holds. We can further bound this quantity by the outage constraint of user $i$ :

$$
O_{i} \leq \mathcal{F}_{F_{i i}}\left(\frac{1}{\mathrm{CEM}_{i}^{\sigma}}\right) \leq \Omega_{i}
$$

By doing so, the right-hand inequality defines a new constraint on $\mathrm{CEM}_{i}^{\sigma}$. By formulating and solving a new problem considering only these new constraints rather than the originals, we guarantee that the original outage constraints are also met. However, such an approach is suboptimal with an error dependent on the tightness between the upper bound and the real outage probabilities.
In effect, we are mapping outage constraints to average SIR constraints. Taking the right-hand inequality from (14) yields

$$
\begin{aligned}
\operatorname{CEM}_{i}^{\sigma} & \geq \frac{1}{\mathcal{F}_{F_{i i}}^{-1}\left(\Omega_{i}\right)} \\
\overline{S I R}_{i} & \geq \frac{\gamma_{i}^{\text {th }}}{\mathcal{F}_{F_{i i}}^{-1}\left(\Omega_{i}\right)}=\Gamma_{i}^{\text {th }}
\end{aligned}
$$

where $\mathcal{F}_{F_{i i}}^{-1}(\cdot)$ denotes the inverse of the appropriate $\mathrm{CDF}$, and we have redefined the outage-mapped average SIR threshold as

$$
\Gamma_{i}^{\mathrm{th}}=\frac{\gamma_{i}^{\mathrm{th}}}{\mathcal{F}_{F_{i i}}^{-1}\left(\Omega_{i}\right)}
$$

for a general fading distribution, which was first introduced in [20] for the Rayleigh fading environment.

The mapped problem can be formulated into the optimization

$$
\begin{aligned}
& \min _{\mathbf{P} \geq \mathbf{0}} \sum_{i=1}^{K} P_{i} \\
& \text { s.t. } \max _{\mathbf{c}_{i},\left\|\mathbf{c}_{i}\right\|_{2}=1} \overline{S I R}_{i}\left(\mathbf{P}, \mathbf{c}_{i}\right) \geq \Gamma_{i}^{\mathrm{th}}, \quad i=1, \ldots, K
\end{aligned}
$$

and there exists a known iterative algorithm to find the optimal solution [4], [20]. We refer to this algorithm as the MMSE-PCA, since the inner optimization utilizes an "average" MMSE receiver to maximize the $\overline{\mathrm{SIR}}_{i}$.

Remark: There may be many circumstances where the upper bound does not hold. For example, we may consider Rician fading as being well approximated by a Nakagami- $m$ model for $m>1$, values that we have already established for which the bound does not hold. In general, all is not lost. First, the optimal scheme of Section IV still holds as it does not rely on any concavity assumptions on the fading CDF. Second, and more importantly, we can view the expression (11) as being an approximate relation rather than an inequality. This implies that the map can no longer guarantee that the original constraints will be met at the solution of the mapped problem. However, should the approximation be accurate, the mapped optimization problems (16) above will result in solutions that are near-optimal, with user outage probabilities being close to their respective targets. Our numerical studies in Section VI validate this claim for $m=\{2,3\}>1$.

\section{E. Examples}

We now show by example how the outage-mapped average SIR thresholds are evaluated for the same distributions considered in Section V-C.

1) Rayleigh: The inverse of the $\operatorname{CDF} \mathcal{F}_{Y}^{-1}\left(\Omega_{i}\right)$ is given by the solution $y^{*}$ to the equation $1-e^{-y}=\Omega_{i}$ taken from (12). The solution is given by $y^{*}=-\log \left(1-\Omega_{i}\right)$, where, for notational convenience, we omit the base on the natural logarithm.

Substituting into (15) gives the mapped threshold in closed form as

$$
\Gamma_{i}^{\mathrm{th}}=\frac{\gamma_{i}^{\mathrm{th}}}{\log \left(\frac{1}{1-\Omega_{i}}\right)}
$$




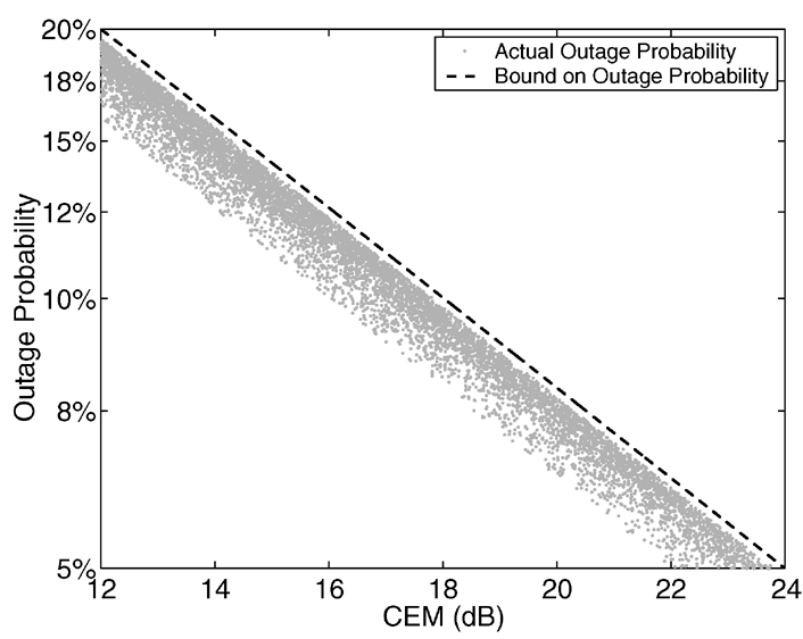

(a)

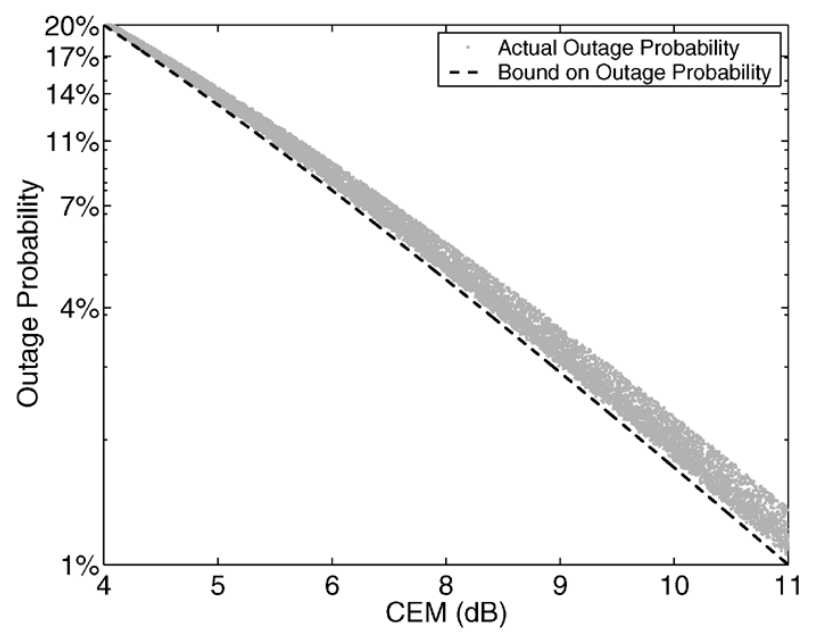

(c)

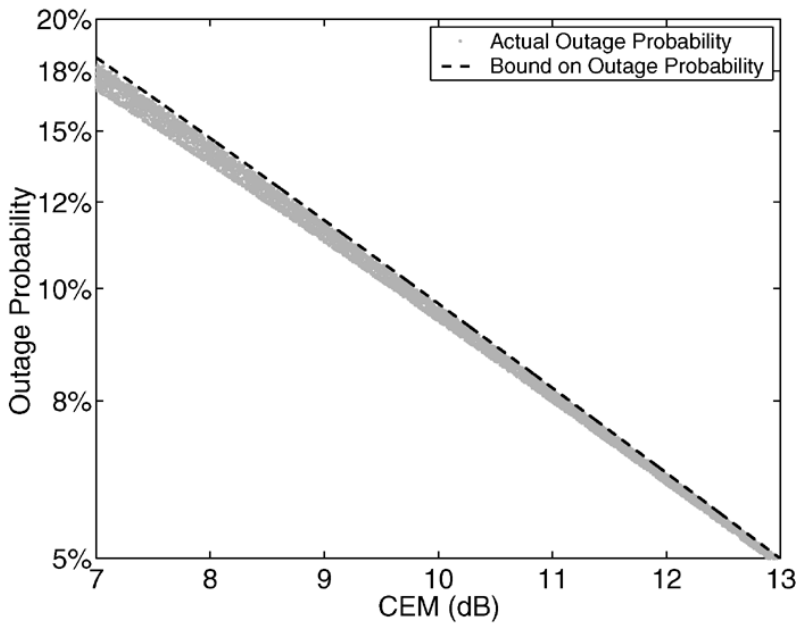

(b)

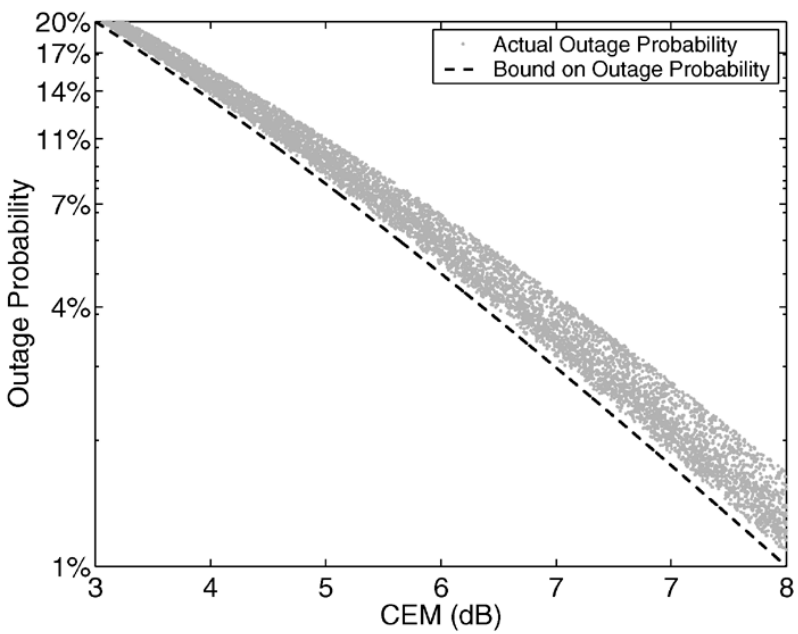

(d)

Fig. 1. CEM, outage probability, and bound for Nakagami- $m$ fading. (a) $m=0.5$. (b) $m=1$. (c) $m=2$. (d) $m=3$.

and is exactly the threshold derived in [20] for the Rayleigh fading environment.

2) Nakagami-m: The inverse of the $\operatorname{CDF} \mathcal{F}_{Z}^{-1}\left(\Omega_{i}\right)$ can be obtained from (13) by finding the solution $\mathrm{CEM}_{i}^{\sigma *}$ satisfying

$$
\Gamma\left(\frac{m}{\mathrm{CEM}_{i}^{\sigma}}, m\right)=\Omega_{i}
$$

This solution can be found by a simple line search. The resulting outage-mapped average SIR threshold is given by

$$
\Gamma_{i}^{\mathrm{th}}=\frac{\gamma_{i}^{\mathrm{th}}}{\mathrm{CEM}_{i}^{\sigma *}}=\gamma_{i}^{\mathrm{th}} \mathrm{CEM}_{i}^{\sigma *}
$$

\section{Simulation Results}

\section{A. Bounds on Outage Probability}

In this subsection, we illustrate the tightness of the bounds of Section V for the Nakagami- $m$ fading distribution. Fig. 1 shows CEM versus outage probability for various fading figures $m$. Each plot was produced by a Monte Carlo simulation having
TABLE I

OUTAGE PROBABILITY BOUND ERROR FOR NAKAGAMI- $m$

\begin{tabular}{|r||r|r|}
\hline \hline$m$ & Mean Error & Standard Deviation \\
\hline \hline$\frac{1}{2}$ & 0.010059 & 0.006604 \\
\hline 1 & 0.002959 & 0.002700 \\
\hline 2 & -0.005006 & 0.002967 \\
\hline 3 & -0.009310 & 0.005520 \\
\hline \hline
\end{tabular}

2000 runs. In each scenario, a random number of users within an isolated cell are generated with random gains $G_{i j}$ and random SIR thresholds $\gamma_{i}^{\text {th }}$. The resulting CEM, outage probability, and associated bound are then computed and plotted. Values outside of the outage probability range of interest $(1 \%-20 \%$ or $5 \%-20 \%$ ) have been discarded.

We clearly see that, for $m \leq 1$, the bound on outage is valid, thus supporting earlier claims. Where the bound does not hold, we may consider (11) as an approximation rather than a bound. This is justified in observing that, for $m$ close to 1 , the mean and standard deviation of the error between the bound and outage probability is reasonably small. Table I quantifies this observation where we note that the error is less than $1 \%$ in the outage probability range of interest, with a reasonably tight spread. 


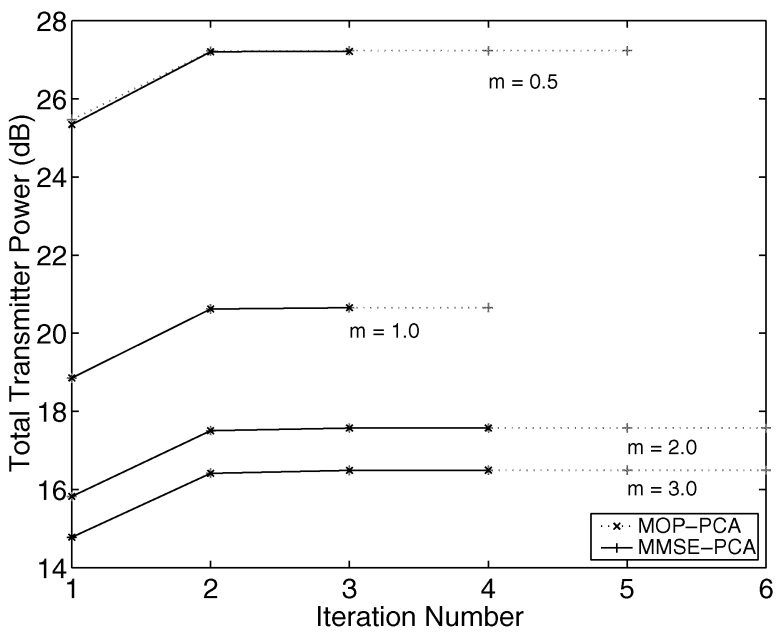

(a)

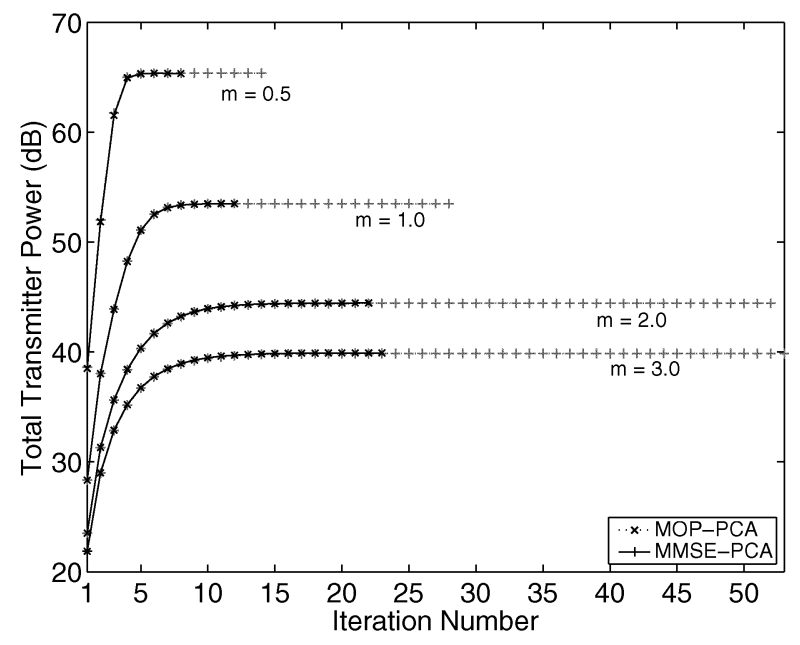

(b)

Fig. 2. Total transmission power for the MOP- and MMSE-PCAs with $N=32$ and Nakagami- $m$ fading. (a) $K=8$ users. (b) $K=32$ users.

\section{B. User Power Optimization With Outage Constraints}

The following simulations consider an isolated circular CDMA cell with radius of $1 \mathrm{~km}$ and uniform location of users within. We model the slowly varying channel gains $G_{i j}$ as a distance-dependent loss (exponent 4) superimposed by log-normal (zero-mean 8-dB variance) shadowing. Once chosen, these gains are fixed. A processing gain $N=32$ is chosen, corresponding to a chip rate of $1.2288 \mathrm{Mc} / \mathrm{s}$ and an encoder input rate of $38.4 \mathrm{~kb} / \mathrm{s}$ under cdma2000 [26]. AWGN receiver power equal to $\sigma^{2}=-130 \mathrm{~dB}$ is also chosen, corresponding to approximately a 1-MHz bandwidth.

User signature sequences are selected at random with elements of $\mathbf{s}_{j}$ taking values $\pm 1 / \sqrt{N}$ with equal probability. Initial receivers $\mathbf{c}_{j}$ are set to the matched filter and initial user powers to the receiver noise power. We employ sequential quadratic programming [27] to numerically minimize the interference function (8) over the receivers at each iteration, whilst the results from [28] are used to numerically compute the outage probability.

We define three QoS classes, each having outage probability and SIR threshold specification pairs $\{(5 \%, 9 \mathrm{~dB}),(10 \%, 8 \mathrm{~dB})$, $(20 \%, 7 \mathrm{~dB})\}$. We assign $25 \%$ of users to the first class, $50 \%$ to the second, and the remaining to the third.

Fig. 2 shows the total sum power of all users as a function of the iteration step, as the Nakagami- $m$ fading figure takes values $m=\{(1 / 2), 1,2,3\}$ identically for all users. A dotted curve represents the MOP-PCA result while the mapped MMSE-PCA result is portrayed as a solid curve.

In all scenarios, the converged total sum powers are almost indistinguishable. For $m \leq 1$, this is due to the tightness of the bound. Where the bound does not apply, we still see an almost indistinguishable result. This is important, as it validates the use of (11) as an approximation of the outage probability and the claims made in Section V-D.

User outage constraints were met with equality (to four significant figures) in all simulations. We illustrate the convergence of the outage probabilities to their final values in Fig. 3, for $K=32$ users and a fading figure of $m=3$, where we have

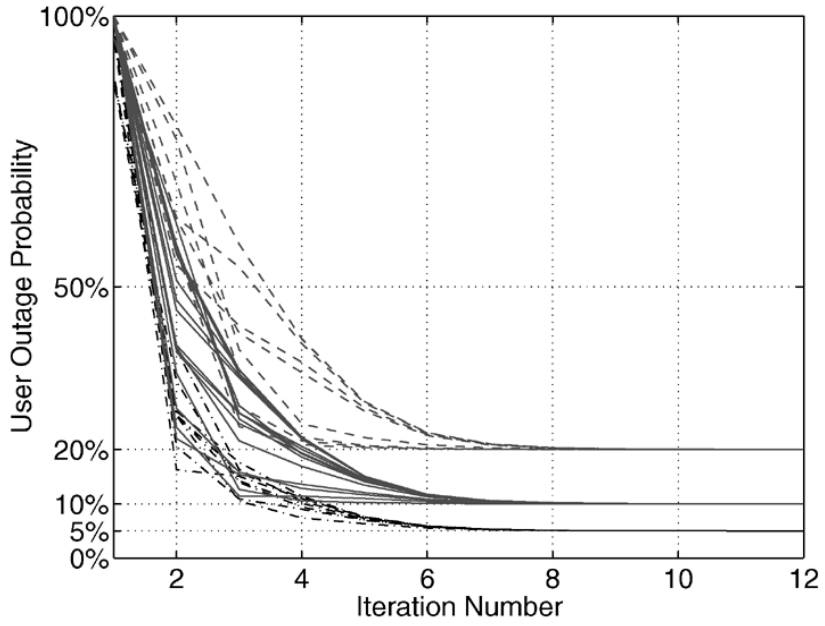

Fig. 3. User outage probability evolution for the MOP-PCA with $K=32$ users, $N=32$, and Nakagami- $m$ fading figure $m=3$. All user outage probabilities reach their target values at convergence.

TABLE II

AVERAGE NORMALIZEd USER POWERS AT CONVERGENCE FOR NAKAGAMI- $m$

\begin{tabular}{|c|c||c|c|c|c|}
\hline \hline \multirow{2}{*}{$m$} & \multicolumn{1}{|c||}{ Algorithm } & \multicolumn{4}{|c|}{$K$} \\
\cline { 3 - 6 } & & 4 & 8 & 16 & 32 \\
\hline \hline \multirow{2}{*}{$\frac{1}{2}$} & MOP & 1.1140 & 1.5369 & 2.4541 & 430.8579 \\
\cline { 2 - 6 } & MMSE & 1.1142 & 1.5408 & 2.4589 & 430.9965 \\
\hline \multirow{2}{*}{1} & MOP & 1.1118 & 1.5199 & 2.4031 & 286.4167 \\
\cline { 2 - 6 } & MMSE & 1.1118 & 1.5199 & 2.4031 & 286.5411 \\
\hline \multirow{2}{*}{2} & MOP & 1.1086 & 1.4901 & 2.3206 & 108.1565 \\
\cline { 2 - 6 } & MMSE & 1.1078 & 1.4900 & 2.3197 & 107.4755 \\
\hline \multirow{2}{*}{3} & MOP & 1.1067 & 1.4732 & 2.2733 & 56.7039 \\
\cline { 2 - 6 } & MMSE & 1.1066 & 1.4730 & 2.2728 & 56.0825 \\
\hline \hline
\end{tabular}

again used the mapped scheme as an approximation to the optimal result. We clearly see the three QoS classes $(5 \%, 10 \%$, and $20 \%$ ) at convergence.

Table II lists average normalized user powers at convergence for a number of scenarios: as the number of users vary as $K=$ $\{4,8,16,32\}$ and the fading figure as $m=\{(1 / 2), 1,2,3\}$. 
Each user's transmit power is first normalized by the power required to meet its outage constraint with equality in a single-user situation,

$$
P_{i}^{*}=\arg \min _{P_{i}}\left[O_{i}\left(P_{i}, \mathbf{c}_{i}\right)-\Omega_{i}\right]^{2}
$$

where the outage probability for user $i$ now only depends on $P_{i}$ and the matched filter $\mathbf{c}_{i}=\mathbf{s}_{i}$ is the optimal receiver. We implement a simple line search to find the solution $P_{i}^{*}$. Second, the average is taken over these normalized powers in each scenario to produce the tabulated results.

Results for the MMSE-PCA are very close to the optimal powers produced by the MOP-PCA, even when $m=\{2,3\}$ and the bound does not apply. In these two cases, the outage probabilities resulting from the MMSE-PCA are slightly higher (above the corresponding outage constraints $\Omega_{i}$ ) than those of the MOP-PCA; however, for practical engineering purposes (to four significant figures), we may still consider the solution to be acceptable.

As one may expect, the average transmission power is greater in more severe fading environments as $m$ decreases. We further observe that for low numbers of users where $K<N$, there exists a reasonably low multiuser power penalty in relation to $K$ as it is scaled upwards. This is to be expected, as the processing gain is sufficiently high to provide a large number of degrees of freedom in the system; the multiuser power penalty being a noise enhancement analogous to that of the decorrelating receiver [2]. As we load the system further to $K=N$, an exponential increase in the average power is observed.

\section{CONCLUSION}

This paper has introduced a new framework for solving power control problems incorporating outage probability constraints. We have presented a general method for creating iterative algorithms with proven convergence to the optimal solution, with only modest conditions on the associated fading distribution. Importantly, users need not be modeled by an identical fading distribution, allowing for a more diverse and realistic cell model. The framework was further developed to solve an extended problem involving a joint optimization of user powers and linear receivers.

New generalized bounds on outage probability were developed for fading distributions having a concave CDF. Such bounds allowed a mapping from outage to average SIR constraints, facilitating a suboptimal scheme to solve the original problem. The error of this suboptimal scheme was found to be exceptionally small for the simulations considered. Where the CDF was not concave, the same method was found to give an excellent approximation to the optimal solution.

There exists two main open research problems and direct extensions that arise from the work presented in this paper:

- an extension toward $L$-branch diversity channels where either multiple antenna configurations or RAKE reception is employed for frequency-selective environments;

- a stochastic interpretation of measured parameters (e.g., slowly varying channel gains $G_{i j}$ ) that are input to the power control algorithms, along the lines of [17].

These are currently under investigation.

\section{REFERENCES}

[1] S. Verdú, Multiuser Detection. Cambridge, U.K.: Cambridge Univ. Press, 1998.

[2] _ - "Minimum probability of error for asynchronous Gaussian multiple-access channels," IEEE Trans. Inf. Theory, vol. IT-32, no. 1, pp. 85-96, Jan. 1986.

[3] U. Madhow and M. L. Honig, "MMSE interference suppression for direct-sequence spread-spectrum CDMA," IEEE Trans. Commun., vol. 42, no. 12, pp. 3178-3188, Dec. 1994.

[4] S. Ulukus and R. D. Yates, "Adaptive power control and MMSE interference suppression," Wireless Networks, vol. 4, Special Issue on Multiuser Detection in Wireless Communications, no. 6, pp. 489-496, 1997.

[5] A. F. Almutairi, S. L. Miller, H. A. Latchman, and T. F. Wong, "Power control algorithm for MMSE receiver based CDMA systems," IEEE Commun. Lett., vol. 4, no. 11, pp. 346-348, Nov. 2000.

[6] A. Yener, R. D. Yates, and S. Ulukus, "Interference management for CDMA systems through power control, multiuser detection, and beamforming," IEEE Trans. Commun., vol. 49, no. 7, pp. 1227-1239, Jul. 2001.

[7] J. Aein, "Power balancing in system employing frequency reuse," COMSAT Tech. Rev., vol. 3, no. 2, pp. 277-300, 1973.

[8] R. Nettleton and H. Alavi, "Power control for a spread spectrum radio system," in Proc. IEEE Vehic. Technol. Conf., 1983, pp. 242-246.

[9] J. Zander, "Performance of optimum transmitter power control in cellular radio systems," IEEE Trans. Veh. Technol., vol. 41, no. 2, pp. 57-62, Feb. 1992.

[10] S. A. Grandhi, R. Vijayan, D. J. Goodman, and J. Zander, "Centralized power control in cellular radio systems," IEEE Trans. Veh. Technol., vol. 42, no. 11 , pp. 466-468, Nov. 1993.

[11] J. Zander, "Transmitter power control for co-channel interference management in cellular radio systems," in Proc. 4th WINLAB Workshop Third Generation Wireless Inf. Networks, 1993, pp. 1281-1285.

[12] D. Mitra, "An asynchronous distributed algorithm for power control in cellular radio systems," in Proc. 4th WINLAB Workshop Third Generation Wireless Inf. Networks, 1993, pp. 249-259.

[13] G. J. Foschini and Z. Miljanic, "A simple distributed autonomous power control algorithm and its convergence," IEEE Trans. Veh. Technol., vol. 43, no. , pp. 541-646, 1993.

[14] S. Grandhi, J. Zander, and R. Yates, "Constrained power control," Int. J. Wireless Pers. Commun., vol. 1, no. 4, pp. 257-270, 1994.

[15] S. V. Hanly, "An algorithm of combined cell-site selection and power control to maximize cellular spread spectrum capacity," IEEE J. Sel. Areas Commun., vol. 13, no. 9, pp. 1332-1340, Sep. 1995.

[16] R. D. Yates and C. Y. Huang, "Integrated power control and base station assignment," IEEE Trans. Veh. Technol., vol. 44, no. 3, pp. 638-644, Mar. 1995.

[17] S. Ulukus and R. D. Yates, "Stochastic power control for cellular radio systems," IEEE Trans. Commun., vol. 46, no. 6, pp. 784-798, Jun. 1998.

[18] N. Bui and S. Dey, "Optimal power control in CDMA over Markov fading channels," in Proc. IEEE Int. Symp. Inf. Theory, Lausanne, Switzerland, Jul. 2002, p. 79.

[19] S. Kandukuri and S. Boyd, "Optimal power control in interference-limited fading wireless channels with outage-probability specifications," IEEE Trans. Wireless Commun., vol. 1, no. 1, pp. 46-55, Jan. 2002.

[20] J. Papandriopoulos, J. Evans, and S. Dey, "Optimal power control for Rayleigh-faded multiuser systems with outage constraints," IEEE Trans. Wireless Commun., vol. 4, no. 11, pp. 2705-2715, Nov. 2005.

[21] Q. T. Zhang, "Outage probability of cellular mobile radio in the presence of multiple Nakagami interferes with arbitrary fading parameters," IEEE Trans. Veh. Technol., vol. 44, no. 8, pp. 661-667, Aug. 1995.

[22] S. Ross, A First Course in Probability, 5th ed. Upper Saddle River, NJ: Prentice-Hall, 1998.

[23] R. D. Yates, "A framework for uplink power control in cellular radio systems," IEEE J. Sel. Areas Commun., vol. 13, no. 7, pp. 1341-1347, Jul. 1995.

[24] M. Nakagami, "The $m$-distribution-A general formula of intensity distribution of rapid fading," in Statistical Methods in Radio Wave Propagation. New York, NY: Pergamon, 1960, pp. 3-36.

[25] H. Suzuki, "A statistical model for urban radio propagation," IEEE Trans. Commun., vol. COM-25, pp. 673-680, Jul. 1977.

[26] Working Document Toward Submission of RTT Candidate to ITU-R, IMT-2000 Process 'The cdma2000 RTT Candidate Submission' ITU-R Draft Document TR45.5, Jun. 1998.

[27] R. Fletcher, Practical Methods of Optimization. New York, NY: Wiley, 1980.

[28] C. T. A. Annamalai and V. K. Bhargava, "Simple and accurate methods for outage analysis in cellular mobile radio systems-A unified approach," IEEE Trans. Commun., vol. 49, no. 2, pp. 303-316, Feb. 2001. 


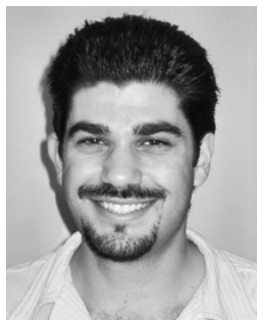

John Papandriopoulos ( $\left.\mathrm{S}^{\prime} 01\right)$ was born in Melbourne, Australia, in 1978. He received the combined B.E. degree in communications engineering and B.App.Sci. degree in computer science from the Royal Melbourne Institute of Technology (RMIT University), Melbourne, Australia, in 2001, and is currently working toward the Ph.D. degree in the Department of Electrical and Electronic Engineering, University of Melbourne, Melbourne, Australia.

His research interests include nonlinear (non)convex optimization techniques and applications, particularly in the cross-layer design of wireless networks and resource allocation in CDMA and OFDM-based networks. He has worked with Telstra, Agilent Technologies, and the 3G Mobile Research and Development Division of NEC Australia.

Mr. Papandriopoulos has served two years as the Chairperson of the University of Melbourne IEEE Student Branch from 2003 to 2005, and he was the recipient of the J. N. McNicol Prize (University Medal) in 2002.

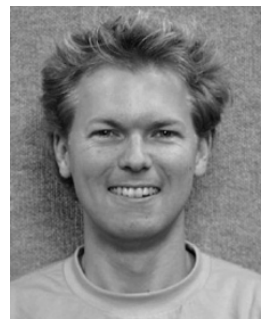

Jamie Evans (S'93-M'98) was born in Newcastle, Australia, in 1970. He received the B.S. degree in physics and the B.E. degree in computer engineering from the University of Newcastle, Newcastle, Australia, in 1992 and 1993, respectively, and the M.S. and $\mathrm{Ph} . \mathrm{D}$. degrees from the University of Melbourne, Australia, in 1996 and 1998, respectively, both in electrical engineering.

From March 1998 to June 1999, he was a Visiting Researcher with the Department of Electrical Engineering and Computer Science, University of California, Berkeley. He returned to Australia to take up a position as Lecturer at the University of Sydney, Sydney, Australia, where he stayed until July 2001. Since that time, he has been with the Department of Electrical and Electronic Engi- neering, University of Melbourne, where he is now an Associate Professor. His research interests are in communications theory, information theory, and statistical signal processing with current focus on wireless communications networks.

Dr. Evans was the recipient of the University Medal of the University of Newcastle and the Chancellor's Prize for Excellence for his Ph.D. dissertation.

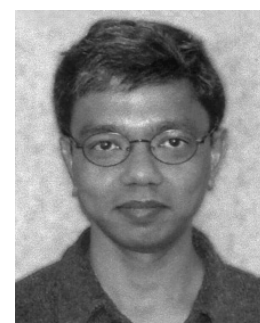

Subhrakanti Dey (M'96-SM'06) was born in Calcutta, India, in 1968. He received the B.Tech. and M.Tech. degrees from the Department of Electronics and Electrical Communication Engineering, Indian Institute of Technology, Kharagpur, India, in 1991 and 1993, respectively, and the Ph.D. degree from the Department of Systems Engineering, Research School of Information Sciences and Engineering, Australian National University, Canberra, Australia, in 1996

He has been with the Department of Electrical and Electronic Engineering, University of Melbourne, Melbourne, Australia, since February 2000, first as a Senior Lecturer and then as an Associate Professor. From September 1995 to September 1997 and September 1998 to February 2000, he was a Postdoctoral Research Fellow with the Department of Systems Engineering, Australian National University. From September 1997 to September 1998, he was a Postdoctoral Research Associate with the Institute for Systems Research, University of Maryland at College Park. His current research interests include signal processing for telecommunications, wireless communications and networks, performance analysis of communication networks, stochastic and adaptive estimation and control, and statistical and adaptive signal processing. He currently serves on the Editorial Board of Elsevier Systems and Control Letters.

Dr. Dey currently serves on the Editorial Board of the IEEE TRANSACTIONS ON AUTOMATIC CONTROL. 